\title{
Charge asymmetry in pure vibrational states of the HD molecule
}

\author{
Sergiy Bubin, ${ }^{1,2}$ Filip Leonarski, ${ }^{2}$ Monika Stanke, ${ }^{3}$ and Ludwik Adamowicz ${ }^{2,4, a)}$ \\ ${ }^{1}$ Quantum Chemistry Research Institute, Kyodai Katsura Venture Plaza 106, Goryo Oohara 1-36, \\ Nishikyo-ku, Kyoto 615-8245, Japan \\ ${ }^{2}$ Department of Chemistry, University of Arizona, Tucson, Arizona 85721, USA \\ ${ }^{3}$ Department of Physics, Nicholas Copernicus University, ul. Grudziadzka 5, PL 87-100, Toruń, Poland \\ ${ }^{4}$ Department of Physics, University of Arizona, Tucson, Arizona 85721, USA
}

(Received 8 December 2008; accepted 11 February 2009; published online 27 March 2009)

\begin{abstract}
Very accurate variational calculations of all rotationless states (also called pure vibrational states) of the HD molecule have been performed within the framework that does not assume the BornOppenheimer (BO) approximation. The non-BO wave functions of the states describing the internal motion of the proton, the deuteron, and the two electrons were expanded in terms of one-center explicitly correlated Gaussian functions multiplied by even powers of the internuclear distance. Up to 6000 functions were used for each state. Both linear and nonlinear parameters of the wave functions of all 18 states were optimized with a procedure that employs the analytical gradient of the energy with respect to the nonlinear parameters of the Gaussians. These wave functions were used to calculate expectation values of the interparticle distances and some other related quantities. The results allow elucidation of the charge asymmetry in $\mathrm{HD}$ as a function of the vibrational excitation. (C) 2009 American Institute of Physics. [DOI: 10.1063/1.3094047]
\end{abstract}

\section{INTRODUCTION}

HD is an interesting model system for very accurate quantum-mechanical calculations because it has slight asymmetry of the electronic charge distribution that originates from the unequal masses of the nuclei. This effect can only be described in a quantum mechanical calculation of this system if the Born-Oppenheimer (BO) approximation is not used. Due to the larger mass of the deuteron than the mass of the proton, the electrons in HD approach the former in average slightly closer than the latter. In a hydrogen atom the average proton-electron distance is $1.0005456 \mathrm{bohr}$ and in the deuterium atom the deuteron-electron distance is 1.0002724 bohr. The asymmetry in the electron behavior near the deuterium and hydrogen nuclei results in an appearance of a small dipole moment in HD. Due to this dipole moment, pure rotational transitions should be visible in the HD experimental spectrum, although they are likely to be very weak. We have studied the HD dipole moment in the ground state in one of our previous works ${ }^{1}$ and the dipolemoment value obtained there agreed very well with the experimental value of $0.000345 \pm 0.1$ a.u. $^{2}$

Non-BO calculations of molecular systems are considerably more difficult than electronic structure calculations based on the BO approximation with the nuclei placed in fixed positions. This is because in such non-BO calculations not only the electronic correlation effects need to be very accurately described but also the correlation effects due to the coupled motions of the electrons and the nuclei as well as the nucleus-nucleus correlated motion must be represented with similar accuracy. The nucleus-nucleus correlation is more difficult to describe than the electron-electron correla-

${ }^{a)}$ Electronic mail: ludwik@u.arizona.edu. tion because the much heavier nuclei avoid each other much more than the electrons. In the past decade we have developed methods for performing non-BO calculations of light atomic and molecular systems. ${ }^{3-8}$ In the calculations we have used several different explicitly correlated Gaussian basis sets. In the calculations of pure vibrational states of diatomic molecules, we used spherically symmetric, explicitly correlated $N$-particle Gaussians multiplied by powers of the internuclear distance. We showed that this type of basis very effectively describes the correlation effects in molecular systems consisting of two nuclei and a few $\sigma$ electrons such as HD. The ground state of the HD molecule was a focus of our earlier non-BO calculations where we used "only" 512 explicitly correlated Gaussian functions in expanding the wave function. ${ }^{9}$ Recently we also reported calculations of the ground and the first excited states of HD performed with 10000 correlated Gaussians. ${ }^{10}$ In the present work we show non-BO calculations for all 18 pure vibrational states of HD performed with 6000 basis functions for each state. The focus of the work is to elucidate the charge asymmetry in HD as a function of the vibrational excitation.

In the non-BO approach, we use a nonrelativistic internal Hamiltonian for the system obtained by rigorously separating the center-of-mass motion from the internal motion. Such a Hamiltonian is isotropic (i.e., rotationally invariant) and its eigenfunctions describing the state of the system transform according to the irreducible representations of the fully symmetric group of rotations. In particular, the wave function of the ground state or any rotationless state of HD is a spherically symmetric s-type wave function. That is why in our calculations we use spherically symmetric explicitly correlated functions (ECFs). Multiplying the ECFs by powers of 
TABLE I. The convergence of the total nonrelativistic non-BO energies $\left(E_{\text {nrel }}\right)$ of the two lowest vibrational states of the HD molecule with the number of basis functions (in hartrees).

\begin{tabular}{cccccc}
\hline \hline & \multicolumn{5}{c}{ No. of basis function } \\
\cline { 2 - 6 }$v$ & 2000 & 3000 & 4000 & 5000 & 6000 \\
\hline 0 & -1.1654718954 & -1.1654719119 & -1.1654719166 & -1.1654719185 & -1.1654719197 \\
1 & -1.1489224960 & -1.1489225611 & -1.1489225754 & -1.1489225810 & -1.1489225845 \\
2 & -1.1331815250 & -1.1331816729 & -1.1331817055 & -1.1331817180 & -1.1331817241 \\
3 & -1.1182331205 & -1.1182334049 & -1.1182334601 & -1.1182334806 & -1.1182334896 \\
4 & -1.1040659876 & -1.1040664499 & -1.1040665434 & -1.1040665905 & -1.1040666237 \\
5 & -1.0906734654 & -1.0906742845 & -1.0906745004 & -1.0906747912 & -1.0906748334 \\
6 & -1.0780558002 & -1.0780569708 & -1.0780571814 & -1.0780572583 & -1.0780572868 \\
7 & -1.0662171804 & -1.0662188005 & -1.0662191177 & -1.0662192349 & -1.0662192819 \\
8 & -1.0551702564 & -1.0551725288 & -1.0551729287 & -1.0551730770 & -1.0551731342 \\
9 & -1.0449358811 & -1.0449387056 & -1.0449392811 & -1.0449394714 & -1.0449395493 \\
10 & -1.0355440344 & -1.0355479732 & -1.0355487022 & -1.0355489733 & -1.0355490755 \\
11 & -1.0270385018 & -1.0270431291 & -1.0270441035 & -1.0270444339 & -1.0270445705 \\
12 & -1.0194754006 & -1.0194820261 & -1.0194834434 & -1.0194838869 & -1.0194840630 \\
13 & -1.0129348937 & -1.0129424990 & -1.0129442943 & -1.0129448378 & -1.0129450477 \\
14 & -1.0075185302 & -1.0075274066 & -1.0075293976 & -1.0075300392 & -1.0075302980 \\
15 & -1.0033639411 & -1.0033733396 & -1.0033752168 & -1.0033758274 & -1.0033760654 \\
16 & -1.0006533204 & -1.0006609924 & -1.0006625007 & -1.0006630111 & -1.0006632170 \\
17 & -0.9996052356 & -0.9996074965 & -0.9996079730 & -0.9996081529 & -0.9996082235 \\
\hline \hline & & & & &
\end{tabular}

the internuclear distance does not change their symmetry, but allows better description of the radial oscillations of the wave function in excited vibrational states.

In the first part of this work we describe the method we used (a more complete description of the method can be found in our recent reviews ${ }^{3,4}$ ). The discussion of the results obtained in the calculations is presented in the second part.

\section{THE METHOD}

The subject of this work is the complete pure vibrational spectrum of the HD molecule. The calculations are performed with the variational method applied separately to each state. In the variational minimization, the total internal energy of the state is expressed as the expectation value of the internal nonrelativistic Hamiltonian, $\hat{H}_{\text {nonrel, obtained }}$ from the "laboratory frame" Hamiltonian by separating out the center-of-mass motion. This separation is achieved by switching from the Cartesian laboratory coordinate system to a system where the first three coordinates are the laboratoryframe coordinates of the center of mass and the remaining $3 N-3$ coordinates are internal coordinates that describe the positions of particles 2,3 , and $N$ with respect to particle 1 (usually the heaviest particle in the system). For HD the internal Hamiltonian has the following form:

$$
\begin{aligned}
\hat{H}_{\text {nonrel }}= & -\frac{1}{2}\left(\sum_{i=1}^{3} \frac{1}{\mu_{i}} \nabla_{\mathbf{r}_{i}}^{2}+\sum_{i=1}^{3} \sum_{j \neq i}^{3} \frac{1}{m_{0}} \nabla_{\mathbf{r}_{i}} \cdot \nabla_{\mathbf{r}_{j}}\right) \\
& +\sum_{i=1}^{3} \frac{q_{0} q_{i}}{r_{i}}+\sum_{i<j}^{3} \frac{q_{i} q_{j}}{r_{i j}} .
\end{aligned}
$$

In Eq. (1), $q_{0}=q_{1}=1$ are the charges of the nuclei (the proton and the deuteron) and $q_{2}=q_{3}=-1$ are the electron charges, $\mathbf{r}_{i}$, $i=1,2,3$, are the position vectors of the proton and the two electrons with respect to the deuteron (placed at the center of the internal coordinate system and called the "reference particle"), $\quad r_{i}$ are their lengths, $\quad r_{i j}=\left|\mathbf{r}_{j}-\mathbf{r}_{i}\right|, \quad m_{0}$ $=3670.4829654 m_{e}$ and $m_{1}=1836.15267261 m_{e}$ are the masses of the deuteron and the proton, respectively, $m_{2}$ $=m_{3}=m_{e}=1$ are the electron masses, ${ }^{11}$ and $\mu_{i}=m_{0} m_{i} /\left(m_{0}\right.$ $\left.+m_{i}\right)$ is the reduced mass of particle $i$. More information on the center-of-mass separation and the form of the internal Hamiltonian (1) can be found elsewhere. ${ }^{12,13}$

The spatial part of the HD non-BO wave functions of the pure (rotationless) vibrational states is expanded in terms of one-center spherically symmetric explicitly correlated Gaussians (ECGs) multiplied by even powers $\left(m_{k}\right)$ of the internuclear distance, $r_{1},{ }^{5-8}$

$$
\phi_{k}=r_{1}^{m_{k}} \exp \left[-\mathbf{r}^{\prime}\left(A_{k} \otimes I_{3}\right) \mathbf{r}\right]
$$

where $\mathbf{r}=\left\{\mathbf{r}_{1}^{\prime}, \mathbf{r}_{2}^{\prime}, \mathbf{r}_{3}^{\prime}\right\}$ and " '" denotes the vector (matrix) transposition. In our previous works, we have shown that these functions very effectively describe nonadiabatic, zeroangular-momentum states of diatomic systems with $\sigma$ electrons. The $r_{1}^{m_{k}}$ factors in function (2) generate radial nodes which appear in the wave function when the molecule becomes vibrationally excited. As shown before, ${ }^{5,6}$ limiting the powers of $m_{k}$ in basis function (2) to only even values has very little effect on the energy, but significantly speeds up the calculations, as the algorithms for calculating the Hamiltonian matrix elements are less complicated in this case.

Function (2) is able to describe bound pure vibrational states of the internal Hamiltonian (1) very well for the following reasons. First, as the Hamiltonian (1) is spherically symmetric, its eigenfunctions for pure vibrational states are also fully spherically symmetric functions and can be expressed in terms of function (2). Second, the particles described by the Hamiltonian (1) have negative (the two electrons) and positive (the proton) charges and are either attracted to or repelled from the reference particle (the deu- 

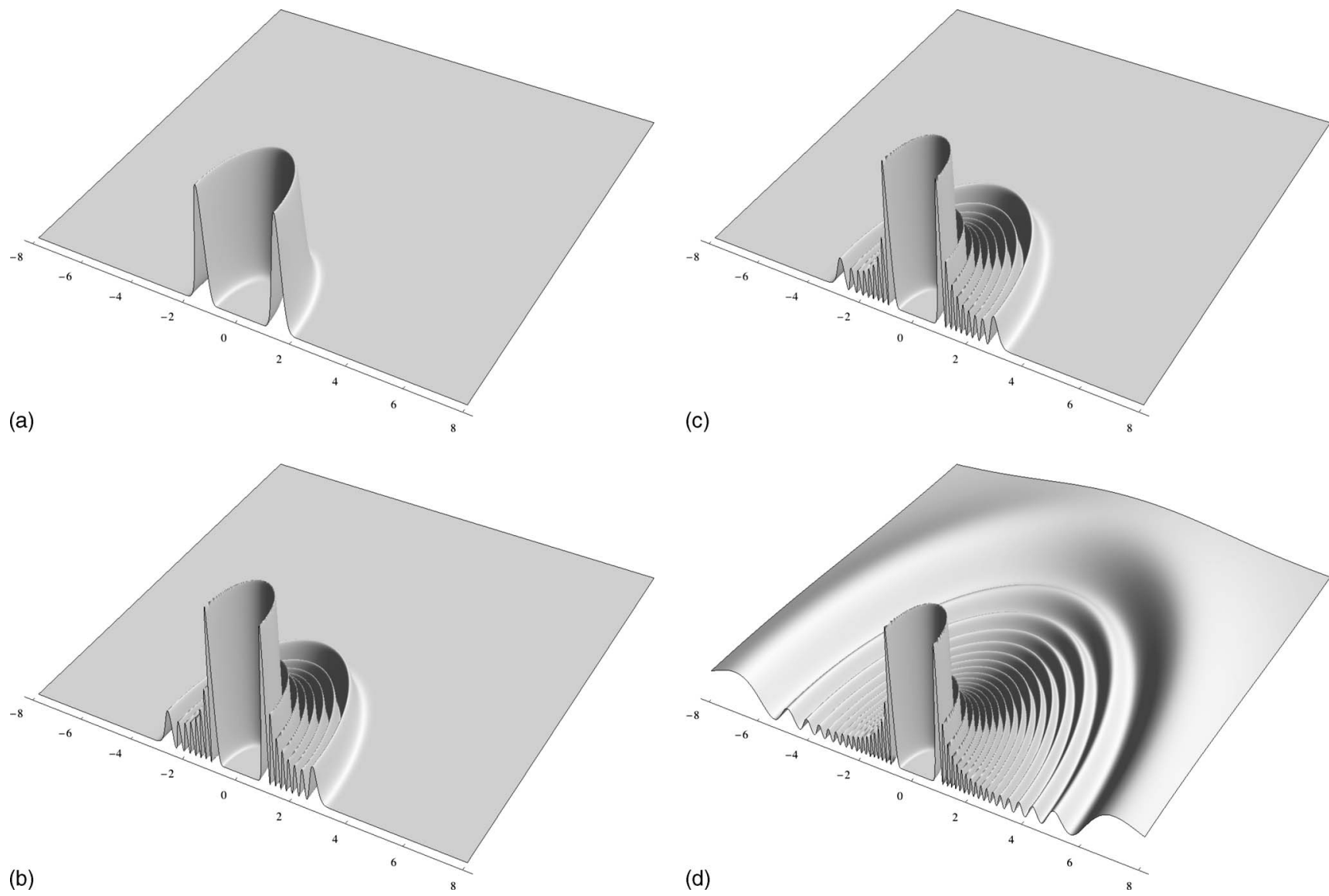

FIG. 1. Deuteron-proton correlation functions $(\mathrm{CF}), g_{i}(\xi)$, for the $v=0,7,8$, and 17 pure vibrational states of the HD molecule. (a) is the $v=0 \mathrm{CF}$, (b) is the $v=7 \mathrm{CF}$, (c) is the $v=8 \mathrm{CF}$, and (d) is the $v=17 \mathrm{CF}$. The densities are shown as two-dimensional functions of the $\xi_{x}$ and $\xi_{y}$ coordinates with the $\xi_{z}$ coordinate set to zero. The $v=0$ plot is done using the $(0,0.14)$ range for the density values, the $v=7$ and $v=8$ plots are done with the $(0,0.07)$ range, and the $v=17$ plot is done with the $(0,0.0015)$ range.

teron) and from themselves. The repulsion between the two electrons can be very well described by Gaussians depending on the interelectron distance in the exponents. Also, while the electron-proton and electron-deuteron attractions can be very well represented by these types of Gaussians, the proton-deuteron repulsion is more difficult to describe because these two particles are much heavier than electrons and they avoid each other to a much higher degree in their relative motion in the molecule. This necessitates the addition of the $r_{1}^{m_{k}}$ factors to the Gaussians, which allows for a much more effective representation of the proton-deuteron separation in the wave function. We refer the reader for more information on the selection of the basis functions for diatomic non-BO calculations to our recent reviews.,

In the present work, the standard variational method is used for calculating the energy and the wave function of a particular state of the system. A separate variational calculation is performed for each state. It involves minimization of the Rayleigh quotient with respect to the linear expansion coefficients, $\left\{c_{k}\right\}$, the Gaussian exponential parameters, $\left\{A_{k}\right\}$, and the pre-exponential powers, $\left\{m_{k}\right\}$,

$$
E=\min \frac{c^{\prime} H\left(\left\{m_{k}\right\},\left\{A_{k}\right\}\right) c}{c^{\prime} S\left(\left\{m_{k}\right\},\left\{A_{k}\right\}\right) c} .
$$

In our approach we used the analytical energy gradient calculated with respect to the Gaussian exponential parameters in the minimization of functional (3). This greatly accelerates the process of the wave function optimization. Also, in order to avoid imposing restrictions on the elements of each $A_{k}$ matrix to make it positive definite and to make the corresponding $\phi_{k}$ basis function square integrable, we used the Cholesky-factored form of $A_{k}, A_{k} \equiv L_{k} L_{k}^{\prime}$, where $L_{k}$ is a lower triangular matrix (all elements above the diagonal are zero). With the Cholesky-factored representation of $A_{k}$, this matrix is automatically positive definite for any real values of the $L_{k}$ matrix elements. In the calculations, the $L_{k}$ matrix elements are the optimization variables, and the analytical energy gradient is calculated with respect to these elements. The preexponential powers, $m_{k}$, in this work ranged from 0 to 250 , and all the powers were partially optimized for each state.

The calculations concern all 18 pure vibrational states of HD. The maximum number of basis functions used for each state was 6000 . This number of functions was generated by growing the basis set for each state from a small randomly selected set of a few dozen functions using a procedure involving successive additions of small groups of functions. When the basis set was relatively small (less than 100 functions) each step involved adding a group of ten functions, one function at a time, optimizing their exponential parameters using the gradient-based minimization approach, and reoptimizing the whole basis set using the gradient-based approach after the addition of the subset was completed. 
TABLE II. Some expectation values calculated for the $v=0,1, \ldots, 17$ pure vibrational states of the HD molecule with the wave functions expanded in terms of 6000 Gaussian basis functions. $r_{1}$ is the proton-deuteron distance, $r_{12}$ is the proton-electron distance, $r_{2}$ is the deuteron-electron distance, and $r_{23}$ is the electron-electron distance. All values are in a.u.

\begin{tabular}{cccccccc}
\hline \hline$v$ & $\left\langle r_{1}^{-1}\right\rangle$ & $\left\langle r_{12}^{-1}\right\rangle$ & $\left\langle r_{2}^{-1}\right\rangle$ & $\left\langle r_{23}^{-1}\right\rangle$ & $\left\langle r_{1}\right\rangle$ & $\left\langle r_{12}\right\rangle$ & $\left\langle r_{2}\right\rangle$ \\
\hline 0 & 0.701604 & 0.903137 & 0.903336 & 0.580391 & 1.442229 & 1.571475 & 1.571185 \\
1 & 0.678247 & 0.885723 & 0.885916 & 0.567188 & 1.525466 & 1.615340 & 1.615045 \\
2 & 0.655416 & 0.868827 & 0.869017 & 0.553909 & 1.611666 & 1.660573 & 1.660274 \\
3 & 0.632995 & 0.852392 & 0.852580 & 0.540481 & 1.701337 & 1.707414 & 1.707110 \\
4 & 0.610856 & 0.836358 & 0.836543 & 0.526813 & 1.795127 & 1.756170 & 1.755863 \\
5 & 0.588858 & 0.820661 & 0.820843 & 0.512800 & 1.893872 & 1.807243 & 1.806932 \\
6 & 0.566838 & 0.805226 & 0.805406 & 0.498313 & 1.998672 & 1.861160 & 1.860847 \\
7 & 0.544602 & 0.789969 & 0.790147 & 0.483192 & 2.110994 & 1.918632 & 1.918317 \\
8 & 0.521916 & 0.774787 & 0.774962 & 0.467236 & 2.232850 & 1.980632 & 1.980316 \\
9 & 0.498483 & 0.759551 & 0.759723 & 0.450187 & 2.367070 & 2.048534 & 2.048219 \\
10 & 0.473920 & 0.744095 & 0.744264 & 0.431700 & 2.517744 & 2.124326 & 2.124013 \\
11 & 0.447707 & 0.728193 & 0.728359 & 0.411308 & 2.691073 & 2.211022 & 2.210713 \\
12 & 0.419115 & 0.711526 & 0.711688 & 0.388344 & 2.896901 & 2.313409 & 2.313107 \\
13 & 0.387065 & 0.693614 & 0.693771 & 0.361813 & 3.152050 & 2.439660 & 2.439368 \\
14 & 0.349818 & 0.673669 & 0.673822 & 0.330104 & 3.488476 & 2.605300 & 2.605021 \\
15 & 0.304222 & 0.650232 & 0.650381 & 0.290250 & 3.978035 & 2.845252 & 2.844992 \\
16 & 0.242954 & 0.619804 & 0.619947 & 0.235220 & 4.847562 & 3.270036 & 3.269800 \\
17 & 0.125229 & 0.562135 & 0.562272 & 0.124370 & 8.990613 & 5.305639 & 5.305431
\end{tabular}

\begin{tabular}{ccccccccc} 
& $\left\langle r_{23}\right\rangle$ & $\left\langle r_{1}^{2}\right\rangle$ & $\left\langle r_{12}^{2}\right\rangle$ & $\left\langle r_{2}^{2}\right\rangle$ & $\left\langle r_{23}^{2}\right\rangle$ & $\left\langle\delta\left(\mathbf{r}_{2}\right)\right\rangle$ & $\left\langle\delta\left(\mathbf{r}_{12}\right)\right\rangle$ & $\left\langle\delta\left(\mathbf{r}_{23}\right)\right\rangle$ \\
\hline 0 & 2.196970 & 2.104322 & 3.131202 & 3.130094 & 5.781954 & 0.226358 & 0.225817 & $1.623741 \times 10^{-2}$ \\
1 & 2.252414 & 2.400880 & 3.319670 & 3.318524 & 6.083447 & 0.220403 & 0.219909 & $1.532194 \times 10^{-2}$ \\
2 & 2.310815 & 2.723569 & 3.520409 & 3.519224 & 6.408235 & 0.214785 & 0.214031 & $1.443487 \times 10^{-2}$ \\
3 & 2.372761 & 3.075939 & 3.735250 & 3.734027 & 6.760769 & 0.209490 & 0.208819 & $1.357086 \times 10^{-2}$ \\
4 & 2.438994 & 3.462531 & 3.966554 & 3.965293 & 7.146758 & 0.204483 & 0.204060 & $1.273480 \times 10^{-2}$ \\
5 & 2.510465 & 3.889253 & 4.217405 & 4.216107 & 7.573627 & 0.199749 & 0.199062 & $1.191085 \times 10^{-2}$ \\
6 & 2.588407 & 4.363964 & 4.491923 & 4.490592 & 8.051202 & 0.195312 & 0.194663 & $1.110056 \times 10^{-2}$ \\
7 & 2.674447 & 4.897328 & 4.795712 & 4.794350 & 8.592702 & 0.191097 & 0.190494 & $1.030321 \times 10^{-2}$ \\
8 & 2.770780 & 5.504243 & 5.136602 & 5.135215 & 9.216355 & 0.187081 & 0.186217 & $9.488991 \times 10^{-3}$ \\
9 & 2.880444 & 6.206153 & 5.525852 & 5.524447 & 9.947977 & 0.183329 & 0.182699 & $8.681950 \times 10^{-3}$ \\
10 & 3.007754 & 7.035020 & 5.980201 & 5.978787 & 10.82533 & 0.179746 & 0.178873 & $7.861124 \times 10^{-3}$ \\
11 & 3.159130 & 8.040927 & 6.525771 & 6.524361 & 11.90626 & 0.176380 & 0.175645 & $6.990626 \times 10^{-3}$ \\
12 & 3.344576 & 9.306869 & 7.205610 & 7.204219 & 13.28436 & 0.173161 & 0.172319 & $6.091455 \times 10^{-3}$ \\
13 & 3.580871 & 10.98207 & 8.096601 & 8.095249 & 15.12360 & 0.170086 & 0.169266 & $5.130368 \times 10^{-3}$ \\
14 & 3.899335 & 13.36841 & 9.353099 & 9.351815 & 17.74700 & 0.167168 & 0.166455 & $4.083583 \times 10^{-3}$ \\
15 & 4.369402 & 17.20322 & 11.34910 & 11.34792 & 21.92345 & 0.164377 & 0.163381 & $2.920116 \times 10^{-3}$ \\
16 & 5.208489 & 25.11561 & 15.40783 & 15.40680 & 30.33132 & 0.161721 & 0.160921 & $1.604434 \times 10^{-3}$ \\
17 & 9.227924 & 88.79661 & 47.38217 & 47.38133 & 94.70291 & 0.159219 & 0.158686 & $1.688639 \times 10^{-4}$ \\
\hline \hline
\end{tabular}

When the number of basis functions exceeded 100, the number of functions added in each step was increased to 20 and the reoptimization of the whole set at the end of each step was done by adjusting the parameters of only one function at a time and cycling over all functions in the basis set.

After the non-BO nonrelativistic wave functions, $\Psi_{i}(\mathbf{r})$, were generated for all eighteen states, they were used to calculate the following expectation values: $\left\langle r_{1}^{-1}\right\rangle,\left\langle r_{12}^{-1}\right\rangle,\left\langle r_{2}^{-1}\right\rangle$, $\left\langle r_{23}^{-1}\right\rangle,\left\langle r_{1}\right\rangle,\left\langle r_{12}\right\rangle,\left\langle r_{2}\right\rangle,\left\langle r_{23}\right\rangle,\left\langle r_{1}^{2}\right\rangle,\left\langle r_{12}^{2}\right\rangle,\left\langle r_{2}^{2}\right\rangle,\left\langle r_{23}^{2}\right\rangle,\left\langle\delta\left(\mathbf{r}_{23}\right)\right\rangle$, $\left\langle\delta\left(\mathbf{r}_{2}\right)\right\rangle$, and $\left\langle\delta\left(\mathbf{r}_{12}\right)\right\rangle$. We also calculated the $\left\langle\delta\left(\mathbf{r}_{1}\right)\right\rangle$ expectation value, but the numbers are very small (in the range of $10^{-10}-10^{-12}$ ) and they are not converged enough to be trusted. The non-BO wave functions were also used to calculate the deuteron-proton correlation function (i.e., the oneparticle relative density of the proton with respect to the deuteron associated with the coordinate $\mathbf{r}_{1}$ ) defined as ${ }^{14}$

$$
\begin{aligned}
g_{i}(\xi) & =\left\langle\Psi_{i}(\mathbf{r})\left|\delta\left(\mathbf{r}_{1}-\xi\right)\right| \Psi_{i}(\mathbf{r})\right\rangle \\
& =\int_{-\infty}^{\infty}\left|\Psi_{i}\left(\xi, \mathbf{r}_{2}, \mathbf{r}_{3}\right)\right|^{2} d \mathbf{r}_{2} d \mathbf{r}_{3},
\end{aligned}
$$

where $\delta\left(\mathbf{r}_{1}-\xi\right)$ is the three-dimensional Dirac delta function. As in the non-BO calculations, both electrons and nuclei are treated on equal footing, the only information one can get on the molecular structure parameters and other related quantities for the particular state of the system is obtained in the form of expectation values of the operators representing these parameters. The nucleus-nucleus correlation functions also provide some information on the structure of the system in different states. This is why we calculate them in this work. 


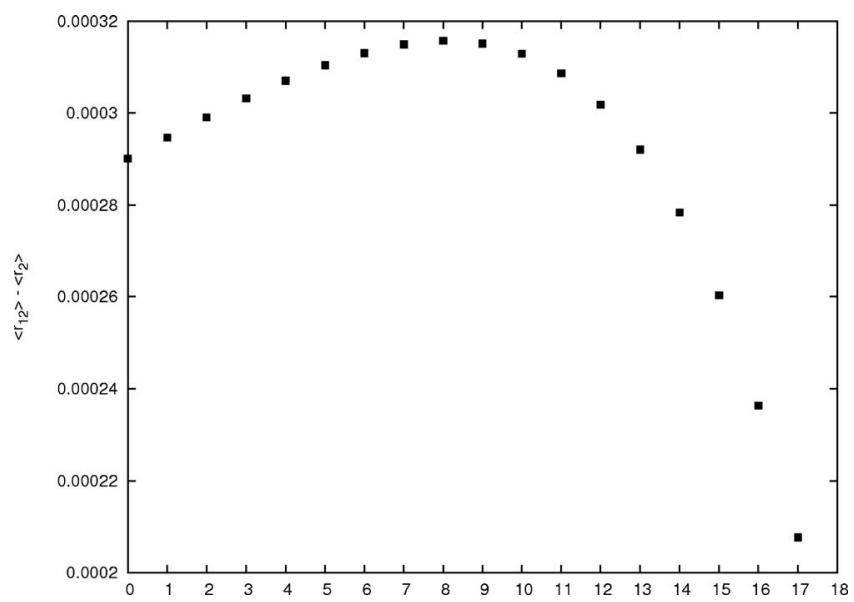

FIG. 2. The difference between the average proton-electron and deuteronelectron distances, $\left\langle r_{12}\right\rangle-\left\langle r_{2}\right\rangle$, calculated with 6000 term wave functions for all eighteen pure vibrational states of the HD molecule.

\section{THE RESULTS}

In Table I we show the total energies for all 18 pure vibrational states obtained in the calculations. For each state, the energy values obtained with basis sets ranging in size from 1000 to 6000 in increments of 1000 are shown. As one can see, the convergence, as expected, is much better for the lowest states than for the highest states. This is related to the increasingly higher oscillatory nature of the wave function as the excitation level increases, which requires a larger number of basis functions in the wave function expansion. The convergence level achieved in the present calculations is quite sufficient for the present results to be by far the most accurate ever obtained for the pure vibrational states of the HD molecule. However, as the energies of the higher states are not converged tight enough yet, the transition frequencies between the consecutive levels could not be calculated with the accuracy equivalent to the experiment. We estimate that it will take approximately 10000 basis functions or more per state to achieve such a sufficient accuracy level. Such calculations will be performed in the near future. In the mean time, the present results are sufficiently accurate to study the charge asymmetry in HD as a function of the vibrational excitation and this is the focus of the present work.

Before we turn to the discussion of the asymmetry of the electron charge distribution in HD, let us first use plots of the proton-deuteron correlation functions for some lowest, intermediate, and highest states to describe the states calculated in this work. As mentioned, the states correspond to pure vibrational excitations. However, as in non-BO molecular calculations of such states the motion of the nuclei couples with the motion of the electrons, the term "vibrational" can only approximately be used to characterize these states because the vibrational and electronic states mix to some small degree in the calculations. A more correct term is "rotationless" because these states correspond to the zero total angular momentum of the system. Due to the mixing, the vibrational quantum number, which is usually associated with the number of nodes in the vibrational part of the wave function, is not, strictly speaking, a good quantum number because there can be small components in the total wave functions where the vibrational parts have different number of nodes than the main component. However, one can still use the vibrational quantum number for ordering the energy levels as we do in this work.

The wave function of each rotationless state of HD is spherically symmetric with respect to the center of the internal coordinate system. Thus, spherically symmetric are also the corresponding deuteron-proton correlation functions. To show this, we present in Fig. 1 the densities for the $v=0,7$, 8 , and 17 states plotted as two-dimensional functions. We could have plotted the correlation functions as onedimensional graphs, but we have chosen two-dimensional representations to better demonstrate the fact that, if the $\mathrm{BO}$ approximations is not assumed, the HD wave functions in the internal coordinate systems are atomlike. The concentric rings on the correlation function plots correspond to the radial maxima of the density function. As one can see, the higher excited states are represented by fast oscillating and more spatially extended functions than the lower states. A wave function with a larger number of oscillations requires more Gaussian functions in the basis set than a less oscillating wave function of a lower state. Also, the $r_{1}^{m_{k}}$ factors in the Gaussians for higher excited states usually have broader power distributions than for the lower states.

The nonrelativistic wave functions obtained for the $v$ $=0,1, \ldots, 17$ states were used to determine the expectation values mentioned before. The values are shown in Table II. Among them there are expectation values of operators dependent on the proton-deuteron distance $\left(r_{1}\right)$, the electron-

TABLE III. Convergence of the difference between the $\left\langle r_{12}\right\rangle$ and $\left\langle r_{2}\right\rangle$ expectation values for the $v=0,1,7,8,16$, and 17 pure vibrational states of the HD molecule with the number of basis functions. All values are in a.u.

\begin{tabular}{cccccc}
\hline \hline & \multicolumn{5}{c}{ No. of basis function } \\
\cline { 2 - 6 }$v$ & 2000 & 3000 & 4000 & 5000 & 6000 \\
\hline 0 & 0.000290 & 0.000290 & 0.000290 & 0.000290 & 0.000290 \\
1 & 0.000295 & 0.000295 & 0.000295 & 0.000295 & 0.000295 \\
$\ldots$ & & & & \\
7 & 0.000315 & 0.000313 & 0.000313 & 0.000313 & 0.000313 \\
8 & 0.000320 & 0.000316 & 0.000316 & 0.000316 & 0.000316 \\
$\ldots$ & & & & \\
16 & 0.000259 & 0.000243 & 0.000238 & 0.000237 & 0.000236 \\
17 & 0.000215 & 0.000209 & 0.000208 & 0.000208 & 0.000208 \\
\hline \hline
\end{tabular}


deuteron distance $\left(r_{2}\right)$, the electron-proton distance $\left(r_{12}\right)$, and the electron-electron distance $\left(r_{23}\right)$. Some of these quantities allow characterization of the asymmetry of the HD electron charge distribution. For example, the difference between the $\left\langle r_{12}\right\rangle$ and $\left\langle r_{2}\right\rangle$ expectation values describes how much closer, on average, an electron approaches the deuteron than the proton in the HD molecule. The difference in the two expectation values are plotted in Fig. 2. Also, in Table III we show how the difference converges with the number of functions in the basis set for some selected lower, intermediate, and higher states. The convergence is quite satisfactory. The $\left(\left\langle r_{12}\right\rangle-\left\langle r_{2}\right\rangle\right)$ difference shows that the charge asymmetry changes with the vibrational excitation and it is the highest for the $v=8$ state and the lowest for the $v=17$ state. The asymmetry change with the vibrational excitation should result in the HD dipole moment also changing. Even though the dipole moment is very small, in relative terms, it may change by as much as $50 \%$ as the molecule de-excites from $v=17$ to $v=8$.

\section{SUMMARY}

In this work we performed very accurate nonrelativistic non-BO calculations of the complete vibrational pure (rotationless) spectrum of the HD molecule. For each of the 18 bound vibrational states the wave function was expanded in terms of up to 6000 explicitly correlated Gaussians. A full optimization of the Gaussian exponential parameters was performed for each expansion. The non-BO wave functions were used to evaluate some expectation values of operators dependent on the deuteron-electron and proton-electron dis- tances and on two-particle contact terms. These expectation values revealed an asymmetry of the electron charge distribution. They also showed that the asymmetry changes with the vibrational excitation. It is the smallest for the lowest and the highest vibrational states and the largest for the intermediate states.

\section{ACKNOWLEDGMENTS}

This work has been supported in part by the National Science Foundation (Contract No. CHE0518610). We also acknowledge partial support of this work by the grant from the Polish Ministry of Science and Higher Education No. N202 041 32/1045. We are grateful to the University of Arizona Center of Computing and Information Technology for providing computer resources for this work.

${ }^{1}$ M. Cafiero and L. Adamowicz, Phys. Rev. Lett. 89, 073001 (2002).

${ }^{2}$ J. B. Nelson and G. C. Tabisz, Phys. Rev. A 28, 2157 (1983).

${ }^{3}$ M. Cafiero, S. Bubin, and L. Adamowicz, Phys. Chem. Chem. Phys. 5, $1491(2003)$

${ }^{4}$ S. Bubin, M. Cafiero, and L. Adamowicz, Adv. Chem. Phys. 131, 377 (2005).

${ }^{5}$ D. B. Kinghorn and L. Adamowicz, J. Chem. Phys. 110, 7166 (1999).

${ }^{6}$ D. B. Kinghorn and L. Adamowicz, Phys. Rev. Lett. 83, 2541 (1999).

${ }^{7}$ S. Bubin and L. Adamowicz, J. Chem. Phys. 118, 3079 (2003).

${ }^{8}$ M. Pavanello, S. Bubin, M. Molski, and L. Adamowicz, J. Chem. Phys. 123, 104306 (2005).

${ }^{9}$ D. B. Kinghorn and L. Adamowicz, J. Chem. Phys. 113, 4203 (2000).

${ }^{10}$ M. Stanke, S. Bubin, M. Molski, and L. Adamowicz, Phys. Rev. A 79, 032507 (2009).

${ }^{11}$ See CODATA 2006 recommended values at physics.nist.gov/cuu.

${ }^{12}$ D. B. Kinghorn and R. D. Poshusta, Phys. Rev. A 47, 3671 (1993).

${ }^{13}$ D. B. Kinghorn and R. D. Poshusta, Int. J. Quantum Chem. 62, 223 (1997).

${ }^{14}$ S. Bubin and L. Adamowicz, Chem. Phys. Lett. 403, 185 (2005). 